\title{
The light elements in the light of 3D hydrodynamical model atmospheres
}

\author{
Martin Asplund \\ Uppsala Astronomiska Observatorium, Box 515, SE-751 20 Uppsala, \\ Sweden
}

\begin{abstract}
The influence of stellar granulation on the line formation of the light elements in metal-poor stars has been investigated by means of 3D hydrodynamical model atmospheres. Due to a lucky (?) coincidence, the effects due to the lower photospheric temperatures with 3D models compared with classical 1D model atmospheres are almost exactly balanced by a pronounced over-ionization of Li I, as concluded from detailed 3D NLTE calculations. Additionally, the effects of convective line asymmetries on ${ }^{6} \mathrm{Li}$ isotope determinations have been investigated and found to be of minor importance.
\end{abstract}

\section{Introduction}

Stellar abundances can function as archaeological remains to trace the cosmic, galactic and stellar evolution and therefore play a central role in astrophysics and cosmology. It is however important to realize that stellar abundances are in fact not observed but rather deduced from an observed spectrum using a multitude of more or less uncertain input data and simplifying assumptions. Atomic data ( $g f$-values, opacities, equation-of-state etc), models of the stellar atmospheres $(1 \mathrm{D}$, hydrostatic and radiative equilibrium, mixing length theory etc), and radiative transfer assumptions (1D, LTE, micro- and macroturbulence etc) are merely a selection of all the necessary ingredients, which may introduce unpleasant systematic errors.

Recently it has become possible to compute realistic 3D, time-dependent, hydrodynamical surface convection simulations of solar-type and metal-poor stars (e.g. Stein \& Nordlund 1998; Asplund et al. 1999) as an alternative to classical 1D hydrostatic model atmospheres, which should remove several of the uncertainties hampering abundance analyses. These 3D model atmospheres are highly successful in reproducing observational diagnostics of the Sun, such as helioseismology, detailed line shapes and asymmetries, and granulation properties (e.g. Stein \& Nordlund 1998; Asplund et al. 2000b). Of particular interest is to apply such models to the line formation of light elements in metal-poor stars, which is discussed here. 


\section{3D hydrodynamical model atmospheres of metal-poor stars}

3D model atmospheres of stellar granulation have been computed with a timedependent, compressible, radiative-hydrodynamics code which solves the equations of mass, momentum and energy conservation (Stein \& Nordlund 1998; Asplund et al. 1999, 2000b). State-of-the-art equation-of-state, which accounts for ionization, excitation and dissociation, and opacities, including the effects of lines, have been used (Mihalas et al. 1988; Gustafsson et al. 1975; Kurucz 1993). In order to obtain a realistic atmospheric structure it is crucial to correctly describe the energy exchange between radiation and gas, which has been included through a simultaneous solution of the 3D LTE radiative transfer. Simulations appropriate for various solar-type stars and metal-poor stars have been performed with physical dimensions covering $\geq 10$ granules at any time. It is noteworthy that the simulations contain no adjustable free parameters besides the stellar parameters, which have been estimated from the IR flux method $\left(T_{\text {eff }}\right)$, Hipparcos parallaxes $(\log g)$ and spectroscopy $([\mathrm{Fe} / \mathrm{H}])$. In particular there are no mixing length parameters involved.

The predictions from the solar simulations agree excellently with observations both in terms of detailed line shapes, asymmetries and shifts, granulation properties, and helioseismological constraints (e.g. Stein \& Nordlund 1998; Asplund et al. 2000b). In particular the line profiles are perfectly matched without invoking any micro- and macroturbulence as a direct consequence of the Doppler shifts introduced by the self-consistent convective velocity fields. The only free parameter in 3D abundance analyses of the Sun is therefore the elemental abundance of the line in consideration, which should result in more secure abundance determinations. For stars, one additionally needs the rotational velocity, which however only affects the line shapes and not the line strengths.

The mean temperature stratification remains close to the radiative equilibrium expectations for solar-metallicity 3D model atmospheres but depart significantly for low-metallicity simulations. The temperature in the line-forming layers is determined mainly by a competition between adiabatic cooling and radiative heating due to absorption of photons in spectral lines. In metal-poor stars there are significantly fewer and weaker lines available which shifts the balance towards lower temperatures since the cooling remains essentially the same (Asplund et al. 1999). As a consequence the temperature in the optically thin layers are much lower than in classical 1D model atmospheres which enforce radiative equilibrium with a difference amounting to as much as $1000 \mathrm{~K}$. Thus, the dependence of the temperature structure on $[\mathrm{Fe} / \mathrm{H}]$ is opposite to that assumed with 1D models.

\section{3D LTE abundances of metal-poor stars}

The drastically different temperature structures in 3D compared with $1 \mathrm{D}$ naturally have a large impact on the line formation and thus on the derived abundances (Asplund et al. 1999). Lines of neutral minority species, low-excitation transitions and strong lines are formed in the higher layers and thus feel the low temperatures there, making them stronger for a given abundance. In particular, the (LTE) Li abundances in metal-poor stars may have been over-estimated by 


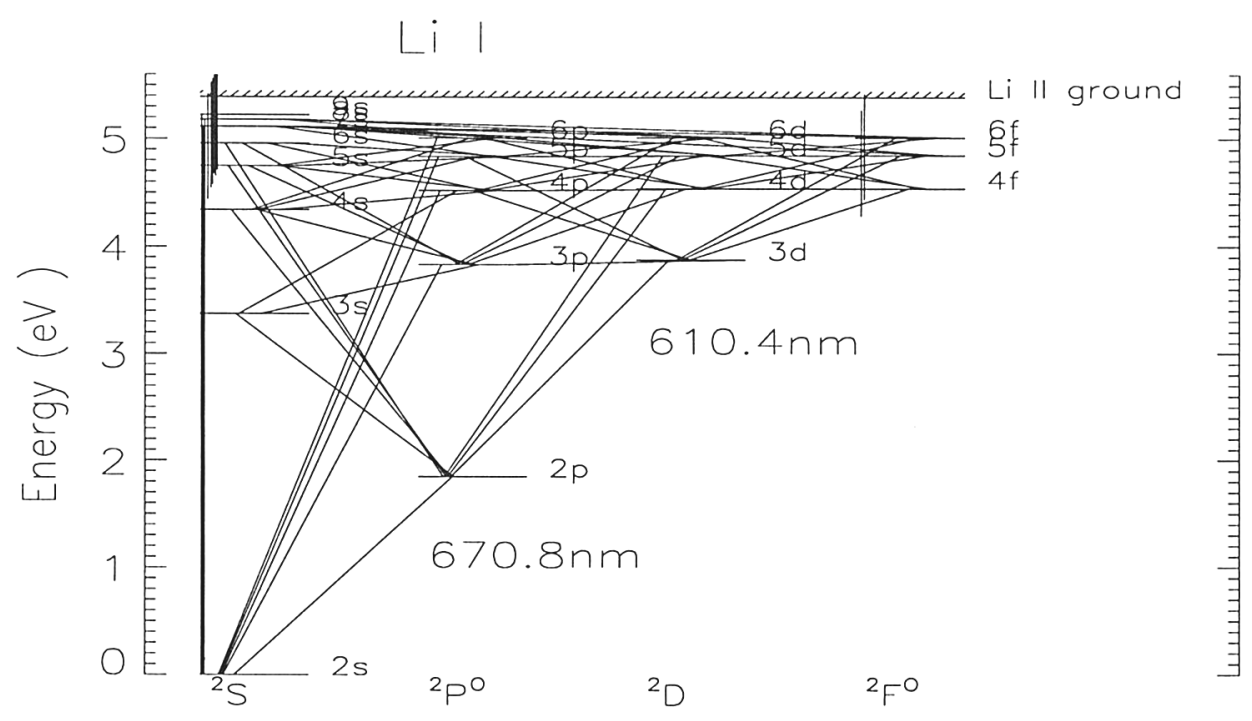

Figure 1. The Li model atom used for the 3D NLTE calculations

0.2-0.35 dex with 1D model atmospheres (but see Sect. 4). Likewise, abundances derived from B I and Fe I lines may be over-estimated by similar amount. On the other hand, Be II, O I and Fe II lines are formed in the deep atmospheric layers, which causes the $1 \mathrm{D}$ abundances to be underestimated by $0.05-0.1$ dex.

The full impact of these LTE results in terms of galactic chemical evolution is impossible to assess here but a few remarks are in order. Although $\mathrm{OH}$ lines have not yet been investigated it is anticipated that the derived $\mathrm{O}$ abundances using $3 \mathrm{D}$ models will decrease relative to with $1 \mathrm{D}$ models, which would make the recently determined $[\mathrm{O} / \mathrm{Fe}]$ vs $[\mathrm{Fe} / \mathrm{H}]$ relation using $\mathrm{OH}$ lines (Israelian et al, these proceedings) flatter. Since the $[\mathrm{Be} / \mathrm{H}]$ vs $[\mathrm{Fe} / \mathrm{H}]$ behaviour should remain essentially unchanged, the $[\mathrm{Be} / \mathrm{H}]$ vs $[\mathrm{O} / \mathrm{H}]$ trend will become slightly shallower, making it more consistent with a primary origin. Furthermore, since B I and Be II react in opposite ways, the $\mathrm{B} / \mathrm{Be}$ ratio may previously have been over-estimated.

It must be remembered, however, that these results are based on the assumption of LTE and thus caution must be exercised. One may worry that the steep temperature gradients may be prone to significant departures from LTE, in particular over-ionization, for species like Li I, B I and Fe I; majority species like $\mathrm{O}$ I and $\mathrm{Fe}$ II should be more immune to such NLTE effects. Indeed Fe I appears to be over-ionized, in which case it seems likely that also Li I would be. Furthermore, spatially resolved spectra of the Sun clearly demonstrates that the LiI line formation process is far from in LTE (Kiselman \& Asplund 2000).

\section{3D NLTE Li I line formation}

In order to investigate possible departures from LTE in the Li I line formation, detailed 3D NLTE calculations have been performed (Asplund \& Carlsson 2000) from a few selected snapshots of the metal-poor convection simulations 


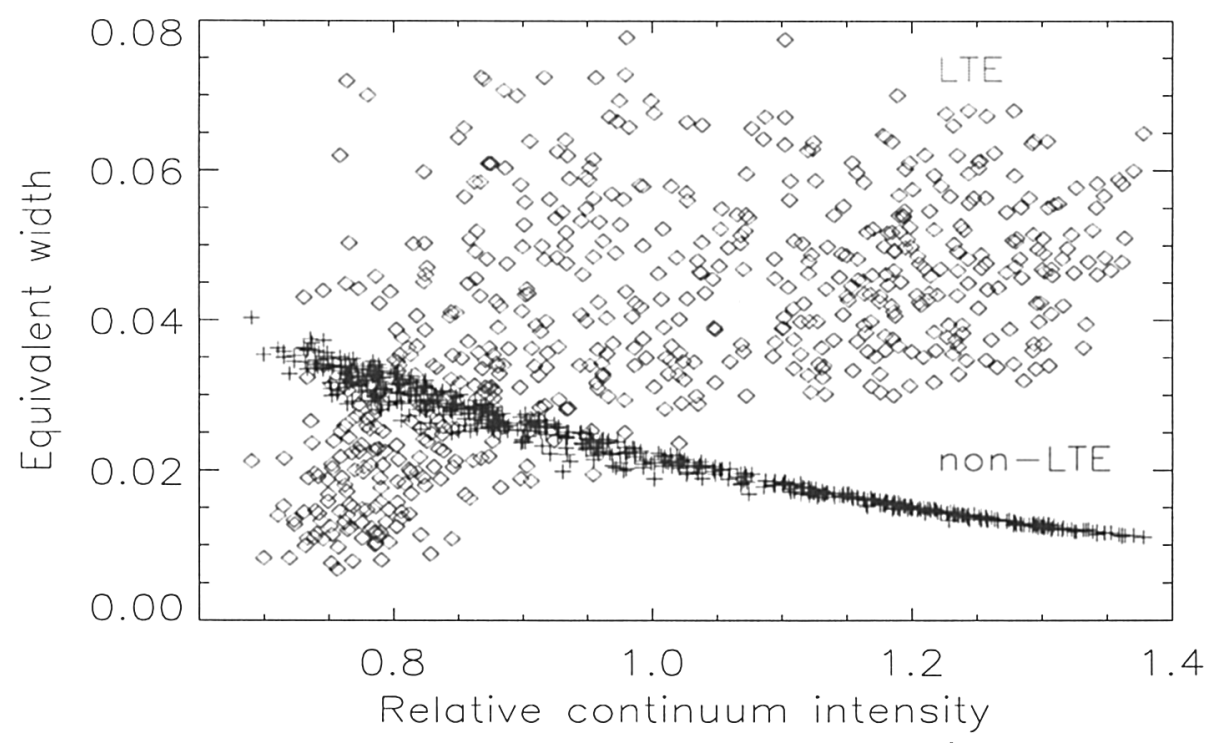

Figure 2. The variation of the LiI $670.8 \mathrm{~nm}$ line strength over the granulation pattern in the HD 140283 simulation in LTE (diamonds) and NLTE (crosses). Notice the remarkably small scatter in NLTE

of HD 140283 and HD 84937 (Asplund et al. 1999) with a 3D-version of the NLTE-code MULTI (Botnen 1997). For the purpose a 21-level Li atom with 70 bound-bound and 20 bound-free radiative transitions (Fig. 1), identical to the one adopted by Carlsson et al. (1994) for 1D NLTE calculations. Additionally similar calculations have been done for the Sun with very similar results to previous investigations (Kiselman 1997).

Similar to the solar case line formation process for the Li I $670.8 \mathrm{~nm}$ line in the 3D metal-poor model atmospheres is far from in LTE, as shown in Fig. 2. In LTE the line strength depend only on the local gas temperature and therefore the line is strong where the temperature is low, i.e. in general over up-flowing regions (high continuum intensities) due to the reversal of the temperature contrast in the optically thin layers (Stein \& Nordlund 1998). As a consequence the LTE line strength tends to increase with the continuum intensity when considering spatially resolved spectra but with a large scatter due to the sensitivity to the local temperature. In contrast without the assumption of LTE the Li I line has the opposite behaviour and show essentially no scatter, reflecting that the line is completely governed by the non-local properties of the radiation field rather than local conditions. The main 3D NLTE effect is clearly over-ionization.

Also when considering spatially averaged profiles there are significant departures from LTE, which in turn translates to large abundance corrections compared with the 3D LTE results. Due to the over-ionization of Li I, the theoretical profile is much weaker than under the assumption of LTE, i.e. the $L i$ abundance in metal-poor stars is larger than estimated with 3D LTE calculations by about 0.3-0.4 dex. Thus the effects of the lower photospheric temperatures in 3D models compared with $1 \mathrm{D}$ models for $\mathrm{Li}$ (Asplund et al. 1999) is almost completely cancelled by departures from LTE, leaving a net "3D effect" (3D 
NLTE - 1D LTE) of $\leq 0.1$ dex for HD 140283 and HD 84937. It must be emphasized however that the findings are still preliminary and that sofar only a couple of snapshots of two metal-poor simulations have been investigated. Considering the two large and partly compensating systematic errors (3D and NLTE) it is much too early to adopt the claimed trend in $\mathrm{Li}$ abundance with $[\mathrm{Fe} / \mathrm{H}]$ from a 1D LTE analysis (Ryan, these proceedings), since the amount of overionization will depend on the stellar parameters. Clearly additional 3D NLTE Li calculations for a range of metallicities and $T_{\text {eff }}$ are urgently needed.

The 3D NLTE calculations presented here have been independently confirmed by Cayrel \& Steffen (these proceedings). They obtain very similar results in spite of their restrictions to 2D model atmospheres and their use of a much smaller Li atom (5 levels). Even if $2 \mathrm{D}$ model atmospheres tend to overestimate the temperature inhomogeneities (Asplund et al. 2000a) compared with 3D the overall conclusions are the same, namely that the Li line formation is determined mainly by the non-local properties of the continuum radiation field.

\section{Lithium isotope ratios in metal-poor stars}

Detections of the ${ }^{6} \mathrm{Li}$ isotope in metal-poor stars help constrain the primordial Li abundance from Big Bang nucleosynthesis, limit the allowed mixing during the stellar lifetime, and provide additional information about cosmic ray spallation of $\mathrm{Li}, \mathrm{Be}$ and $\mathrm{B}$ (Nissen, these proceedings). The determinations of ${ }^{6} \mathrm{Li}$ abundances are based on the increased width and asymmetry of the Li I $670.8 \mathrm{~nm}$ doublet introduced by the isotope shift. Since classical 1D model atmospheres can not predict the inherent convective line asymmetries and must invoke adhoc macroturbulence line broadening, the conclusions suffer from unfortunate uncertainties. An attractive alternative is provided by $3 \mathrm{D}$ model atmospheres which are able to predict the convective line asymmetries, leaving any possible remaining line asymmetry to be attributed to ${ }^{6} \mathrm{Li}$. Furthermore, no ad-hoc extra broadening parameters like micro- and macroturbulence must be specified (Asplund et al. 2000).

In order to verify the claimed detection of ${ }^{6} \mathrm{Li}$ in the metal-poor halo star HD 84937 (Smith et al. 1993), a similar analysis has been performed based on both 1D and 3D model atmospheres using the same observed spectrum. The 3D profiles were averaged over a sufficiently long time-sequence to produce statistically significant results. Due to computing-time considerations departures from LTE were not considered here. The stellar broadening (1D: macroturbulence+rotation, 3D: rotation) was determined from $\mathrm{Ca}$ I and $\mathrm{Fe}$ I lines of similar strength. Due to the large isotope shift in relation to the convective line asymmetries, almost identical results were obtained with $1 \mathrm{D}$ and 3D models: ${ }^{6} \mathrm{Li} /{ }^{7} \mathrm{Li}=0.04 \pm 0.02$, in agreement with previous determinations. This finding thus lends confidence to the reality of the detection of the isotope in HD 84937.

A very high $S / N$ spectrum of the metal-poor halo star G271-162 obtained during the commissioning of UVES on VLT/UT2 has been analysed in a similar fashion with a suitable $3 \mathrm{D}$ model. The Li feature reveals a possible detection of ${ }^{6} \mathrm{Li}$ at the level of ${ }^{6} \mathrm{Li} /{ }^{7} \mathrm{Li}=0.02 \pm 0.01$ (Nissen et al. 2000). The smaller ${ }^{6} \mathrm{Li}$ abundance in G271-162 than in the almost identical HD 84937 may indicate an intrinsic scatter in ${ }^{6} \mathrm{Li} /{ }^{7} \mathrm{Li}$ in the ISM at a given metallicity. 


\section{Concluding remarks}

It is clear from comparison with observations (e.g. helioseismology, granulation properties, line shapes and asymmetries) that 3D hydrodynamical model atmospheres are highly realistic without invoking any free parameters. Abundance analyses based on such 3D models should therefore in general be more reliable than corresponding investigations using classical 1D model atmospheres. In particular for metal-poor stars there are significant differences to 1D models which translate to a large impact on the emergent stellar spectra.

Even though the detailed 3D NLTE results for Li presented here apparently almost completely compensate the effects due to the use of hydrodynamical model atmospheres (Asplund et al. 1999) making them very similar to the 1D LTE predictions, it is important to realize that this is more a lucky coincidence than a general truth. It is by no means correct to say that 3D LTE abundances are erroneous in general, even if it may be true for Li (Cayrel \& Steffen, these proceedings). Lines of other species, like ionized species, high-excitation transitions or very weak lines, are much more immune to departures from LTE, whether with $1 \mathrm{D}$ or $3 \mathrm{D}$ model atmospheres. It should be remembered that $1 \mathrm{D}$ analyses contain additional systematic errors by not taking into account properly the convection and will therefore in general produce less reliable results than 3D LTE investigations. Naturally, however, those results must also be verified by detailed 3D NLTE calculations whenever possible.

The full version of the present talk with additional figures can be found at: http://www.astro.uu.se/ martin/talks/Natal99

Acknowledgments. The author greatly appreciates fruitful collaboration with M. Carlsson, P.E. Nissen, $\AA$. Nordlund, R.F. Stein, and R. Trampedach, without whom the above-mentioned projects would never have been possible.

\section{References}

Asplund, M., \& Carlsson M. 2000, A\&A, submitted

Asplund, M., Nordlund, §., Trampedach, R., \& Stein, R.F. 1999, A\&A 346, L17

Asplund, M., Ludwig, H.-G., Nordlund, §., \& Stein, R.F. 2000a, A\&A, in press Asplund, M., Nordlund, §̊., Trampedach, R., \& et al. 2000b, A\&A, in press Botnen, A.V. 1997, Cand. Sci. thesis, University of Oslo

Carlsson, M., Rutten, R.J., Bruls, J.H.M.J., \& et al. 1994, A\&A, 288, 860

Gustafsson, B., Bell, R.A., Eriksson, K., \& Nordlund, Å. 1975, ApJ, 42, 407

Kiselman, D. 1997, ApJ, 489, L107

Kiselman, D., \& Asplund M. 2000, in: Cool stars, stellar systems and the Sun, 11th Cambridge workshop, in press

Kurucz, R.L. 1993, CD-ROM, private communication

Mihalas, D., Däppen, W., \& Hummer, D.G. 1988, ApJ, 331, 815

Nissen, P.E., Asplund, M., Hill, V., \& D'Odorico, S. 2000, A\&A, in press

Smith, V.V., Lambert, D.L., \& Nissen, P.E. 1993, ApJ, 408, 262

Stein, R.F., \& Nordlund, Å. 1998, ApJ, 499, 914 